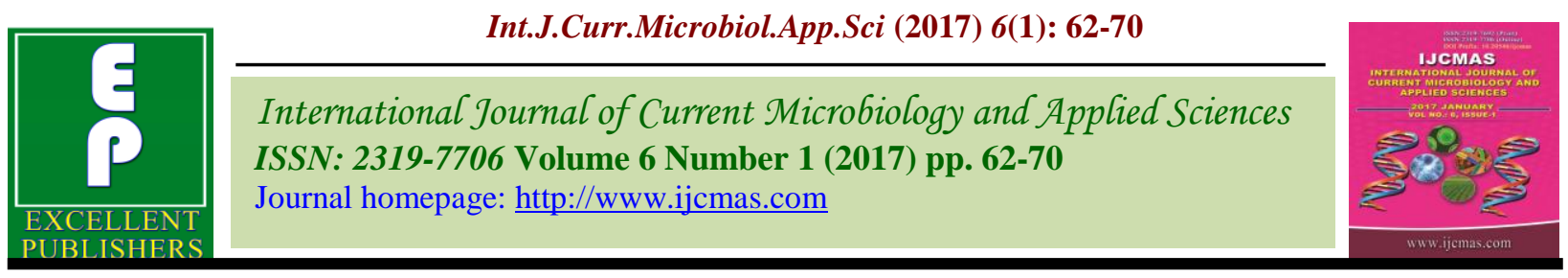

Original Research Article

http://dx.doi.org/10.20546/ijcmas.2017.601.008

\title{
Degradation of Malachite Green (Dye) by using Photo-Catalytic Biogenic Silver Nanoparticles Synthesized using Red Algae (Gracilaria corticata) Aqueous Extract
}

\author{
Subramanian Poornima and Karuppiah Valivittan*
}

Department of Biotechnology, St. Peter's University, Avadi, Chennai-600054, India

*Corresponding author

\begin{tabular}{|c|c|}
\hline & $\mathrm{C} \mathbf{T}$ \\
\hline Keywords & \multirow{3}{*}{$\begin{array}{l}\text { In this paper, we report on biosynthesis of silver nanoparticles using Gracilaria } \\
\text { corticata (Red seaweed) at room temperature along with photo-catalytic } \\
\text { degradation of Malachite green dye. UV spectral analysis showed peak at } 380 \mathrm{~nm} \\
\text { with special reference to the excitation of surfaces plasmon vibration by silver } \\
\text { nanoparticles. The morphology of silver nanoparticles was found to be spherical } \\
\text { and ranges about } 100 \mathrm{~nm} \text { as confirmed by HR-SEM. Positive zeta potential value } \\
\text { of } 7.7 \mathrm{mV} \text { suggests that the nanoparticles are moderately stable in colloidal } \\
\text { solution. Further, photo-catalytic degradation of malachite was measured } \\
\text { spectrophotometrically by using silver as nano-catalyst under visible light } \\
\text { illumination. The results revealed that biosynthesized silver nanoparticles } \\
\text { using Gracilaria corticata was found to be impressive in degrading malachite } \\
\text { green. }\end{array}$} \\
\hline Article Info & \\
\hline & \\
\hline
\end{tabular}

\section{Introduction}

Nanotechnology deals with the synthesis of nanoparticles with controlled size, shape, and dispersity of materials at the nanometer scale length and their potential use for human wellbeing. Nanometer sized materials have a high surface area; and a high fraction of surface atoms have been studied because of their exclusive properties such as optic, electronic, and catalytic. Among all nanoparticles noble metal nanoparticles have enormous applications in diverse areas such as bioimaging, sensor, diagnosis, and novel therapeutic in biomedical field (Salata, 2004). Metallic silver and silver nanoparticles were recently applied as antimicrobial agents in various products such as cosmetics (Kokura et al., 2010), animal feed (Hojberg et al., 2005), Coating of catheters (Roe et al., 2008), wound dressing (Fernandez et al., 2008), and water purification (Choi et al., 2008) with a minimal risk of toxicity in humans. Nowadays the biological systems were eagerly used for nanoscale material synthesis and assembly is an alternative method of physical and chemical process. Green approach of nanoparticles synthesis by biological entities has been gaining great advantages which are environmental benign, less toxic, and time consuming; and also it is a single step process. Currently, plant and plant derived 
materials are used for nanoparticles synthesis which is more compatible than the microbemediated nanoparticles synthesis process because they eliminate the culture maintenance and are easy to handle. Nanoparticles synthesis by medicinal plants shows more benefit; they may enhance the antibacterial activity of silver nanoparticles, because the medicinally valuable active biomolecule present in the plants may bind on the surface of the nanoparticles and reduce the silver ions to silver nanoparticles.

Malachite green (MG) has numerous industrial applications (dyeing of silk, leather, plastics and paper). Their appearance is harmful for humans and animals following inhalation and/or ingestion, produces toxicity to respiratory system and reduces fertility in humans. The triphenylmethane dyes biodegradation due to presence of nitrogen in their back bone (generate carcinogenic, genotoxic, mutagenic and teratogenic problems) is a difficult task (Papinutti et al., 2006). MG has high resistance to light and oxidizing agents, while its removal based on biological treatment and chemical precipitation has low efficiency (Dogan Uluozlu et al., 2008). It is now established that $\mathrm{Ag}$ is considered a promising semiconductor that is extensively involved in removal of several toxic organic containments through both adsorption and photo-catalytic process due to the stability of its chemical structure, biocompatibility, strong oxidizing power, non-toxicity and low cost of the metal precursors. Ag can be only triggered by UV radiation that encompasses only about 4\% $5 \%$ of natural solar radiation due to its wide band gap energy $(3.2 \mathrm{eV})$. This defect limits the industrialization of this powder. Moreover, the mineralization processes through various redox reactions are encountered by the rapid recombination of the charge carriers. Another major defect in the preparation of nano titanium oxide through sol-gel route is the rapid hydrolysis of titanium alkoxide salts that lead to irregular condensation of hydroxide particles into amorphous solid (Ismail, 2012). This random condensation of particles is the primary cause for obtaining irregular particle and pore structure.

The synthesis of AgNPs using plant extracts is simple, economical and requires less reaction time. Moreover, the microorganisms generate nanoparticles at a much slower rate than the plant extracts. Leaf extracts of Ficus benghalensis (Saxena et al., 2012), Crossandra infundibuliformis (Kaviya et al., 2012) have been shown to produce AgNPs with good stability. Other parts of plants such as tuber, bark and buds have also been reported in AgNPs synthesis. In this study, we successfully reported the biosynthesis of silver nanoparticles using Gracilaria corticata aqueous extract. Synthesized silver nanoparticles were applied to dye degradation under sunlight irradiation

\section{Materials and Methods}

\section{Materials}

Silver nitrate (>99\% pure) was purchased from Sigma Aldrich, India. Potato dextrose broth, Potato dextrose agar, Nutrient broth, Nutrient agar plate, was supplied by $\mathrm{Hi}$ media, India.

\section{Sample collection and preparation (Gracilaria corticata)}

The red algae (Gracilaria corticata) were collected from the Rameshwaram sea coast area, Tamilnadu, India and were brought to the nanotechnology laboratory and washed with distilled water several times to remove the impurities. The clean algae were dried at room temperature in the shade for a week and powdered using a mortar and pestle. 
Preparation of Gracilaria corticata (red algae) extract

Dried powdered Gracilaria corticata $(5 \mathrm{~g})$ was mixed with $100 \mathrm{ml}$ distilled water then the solution was kept for continues heating at $80^{\circ} \mathrm{C}$ for 1 hour at room temperature with frequent shaking. After that the extract were filtered by using Whatmann No1 filter paper. The extract was collected and stored at $4^{\circ} \mathrm{C}$ for further use.

Synthesis of silver nanoparticles from Gracilaria corticata

$10 \mathrm{ml}$ of the aqueous extract of Gracilaria corticata was added into $90 \mathrm{ml}$ of aqueous solution of $1 \mathrm{mM}$ Silver nitrate. The mixture was exposed to a range of controlled temperatures for 24h. Appearance of brown color in solution indicated the formation of AgNPs. The solution was then kept in dark for further analysis collected and stored at $4^{\circ} \mathrm{C}$ for further use.

\section{Photo-catalytic malachite green dye degradation}

The photo-catalytic degradation of malachite green was evaluated by biosynthesized $\mathrm{Ag}$ nanoparticles. All the experiments were performed outdoor with sun as the main source of light (Li et al., 2005). Prior to the experiment, a suspension was prepared by adding $20 \mathrm{mg}$ of $\mathrm{Ag}$ nanoparticles to $50 \mathrm{ml}$ of methyl orange solution (Fisher Scientific). Later, the mixture was allowed to stir constantly for about $30 \mathrm{~min}$ in darkness to ensure constant equilibrium of $\mathrm{Ag}$ nanoparticles in the organic solution.

During the reaction, the mixture was kept under sunlight within a Pyrex glass beaker and stirred constantly. The mean temperature was found to be $30^{\circ} \mathrm{C}$ with $10 \mathrm{~h}$ mean shine duration, A control was prepared in similar manner without addition of silver nanoparticles. The absorption spectrum of the suspension mixture was measured periodically using a UV-visible spectrophotometer (Shimadzu, UV-2450, Japan) after centrifugation to ensure the degradation of malachite green solution.

\section{Characterization of Ag nanoparticles}

\section{UV - Visible spectrum for synthesized nanoparticles}

The nanoparticles were monitored by UVvisible spectrum at various time intervals. The UV - Visible spectra of this solution was recorded in spectra 50 ANALYTIKJENA Spectrophotometer, from 250 to $400 \mathrm{~nm}$.

\section{Particle Size and Zeta potential analyzer for synthesized nanoparticles}

The aqueous suspension of the synthesized nanoparticles was filtered through a $0.22 \mu \mathrm{m}$ syringe driven filter unit and the size of the distributed nanoparticles were measured by using the principle of Dynamic Light Scattering (DLS) technique made in a Nanopartica (HORIBA, SZ-100) compact scattering spectrometer.

\section{High Resolution Scanning Electron Microscope (HR-SEM)}

The structural morphological characteristics of the bacterial sample were observed under scanning electron microscope (HR-SEM) Hitachi's SU6600 at magnification ranging from $10 \mathrm{X}$ to $600,000 \mathrm{X}$ operated at accelerating voltage of $30 \mathrm{kv}$.

\section{Results and Discussion}

It is well know that seaweeds are extremely different from terrestrial plants with the ability to reduce silver ions. However, when 
$1 \mathrm{mM} \mathrm{AgNO}_{3}$ solution was added to the aqueous seaweed broth (ASB), no reaction occurred. Instead, after $24 \mathrm{~h}$ of incubation at room temperature, the color of the solution intensified to brown indicating the formation of Ag nanoparticles as shown in (Fig. 1) inset. The characteristic color change obtained may perhaps be due to the excitation of surface plasmon resonance (SPR) and reduction of $\mathrm{AgNO}_{3}$.

\section{UV-Visible spectral analysis}

It is well- known that silver nanoparticles exhibit brown color, which arises due to excitation of surface Plasmon vibrations of the silver nanoparticles. After addition of $1 \mathrm{mM}$ silver nitrate solution to the aqueous extract, the colour of the composition has been changed to dark brown colour. The maximum absorbance peak is observed at $380 \mathrm{~nm}$ for Gracilaria corticata (Fig. 2). The overall observations suggest that the bio reduction of (silver ions) $\mathrm{Ag}^{+}$to $\mathrm{Ag}^{(0)}$ was confirmed by UV-Visible spectroscopy.

\section{Dynamic light scattering analysis}

The particle size distribution spectra for the silver nanoparticles were recorded as diameter (nm) verses frequency $(\% / \mathrm{nm})$ spectra with diameter $(\mathrm{nm})$ on $\mathrm{x}$-axis and frequency $(\% / \mathrm{nm})$ on $y$-axis. The zeta potential spectra for the silver nanoparticles were recorded zeta potential verses intensity spectra with zeta potential $(\mathrm{mV})$ on $\mathrm{x}$-axis and intensity (a.u) on y-axis. Dynamic light scattering technique has been used to measure hydrodynamic diameter of the hydrosol (particle suspension). Gracilaria corticata AgNPs was found to be $96.5 \mathrm{~nm}$ (Fig. 3a) the recorded value of zeta potential of the silver nanoparticles was $7.7 \mathrm{mV}$ (Fig. 3b) which resulted in the agglomerated state of the formed AgNPs

\section{Scanning Electron Microscopy analysis}

The surface morphology, size and shape of Gracilaria corticata silver nanoparticles were characterized from the SEM micrograph, it is evident that AgNPs were spherical in shape and were poly-dispersed. The measured average size of AgNPs was $100 \mathrm{~nm}$ and the nanoparticles range from 100-500nm occasional agglomeration of the AgNPs has been observed. The electrostatic interactions and hydrogen bond between the bioorganic capping molecules bond are responsible for the synthesis of silver nanoparticles using Gracilaria corticata extract (Fig. 4). In this study, small AgNPs were attached to the surface of large bio-molecules. The aggregation of the nanoparticles indicates that they were in the direct contact, but stabilized by a capping agent, It is evident from the micrograph that individual silver nanoparticles as well as a number of aggregates are present and they are spherical in shape

\section{Photo-catalytic degradation studies}

Photo-catalytic degradation of malachite green dye was investigated using biometrically synthesized silver nano particles by solar irradiation technique at different time intervals as shown in (Fig. 5). The characteristic absorption peak of malachite green for Gracilaria corticata solution was found to be $380 \mathrm{~nm}$. Degradation of malachite green was visualized by decrease in peak intensity within $6 \mathrm{~h}$ of incubation time.

There is no considerable shift in peak position for malachite green solution without exposure to Ag nano-catalysts. Kansal et al. (2008) have reported that compared to other irradiation techniques, solar light was found to be faster in decolorizing malachite green in the presence of metal nanoparticles as catalyst. 
Fig.1 Gracilaria corticata

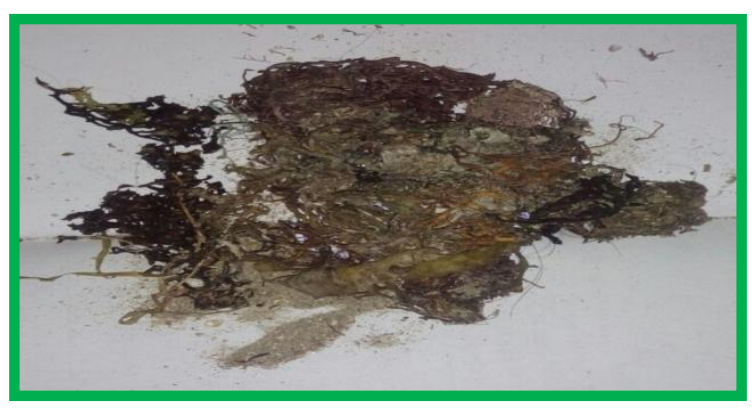

Fig.2 UV-visible spectroscopy showing Gracilaria corticata mediated synthesized silver nanoparticles

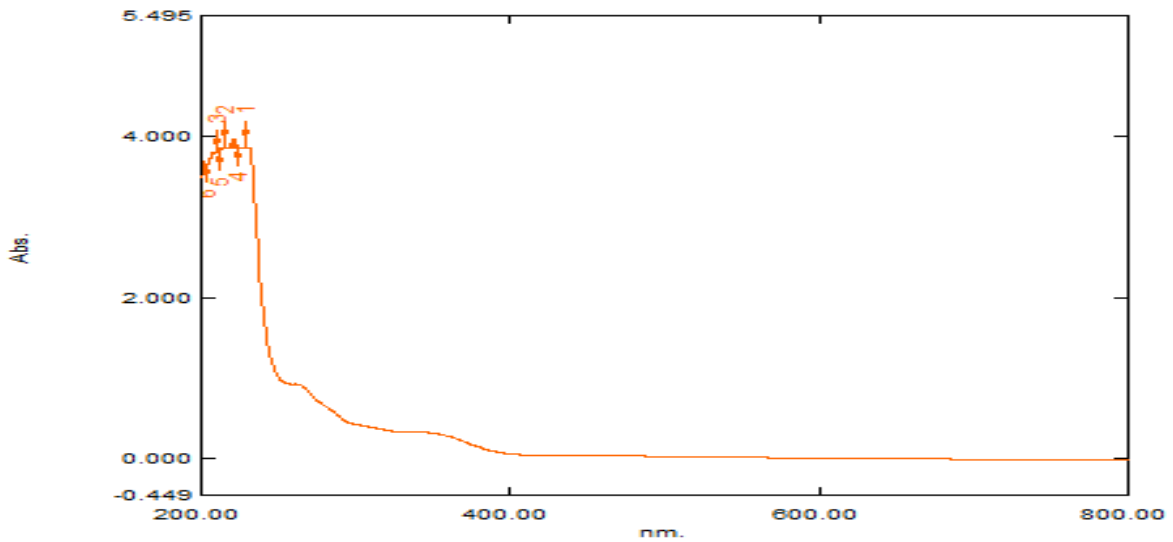

Fig.3 DLS analysis showing a) Particle size b) Zeta potential Gracilaria corticata mediated synthesized silver nanoparticles

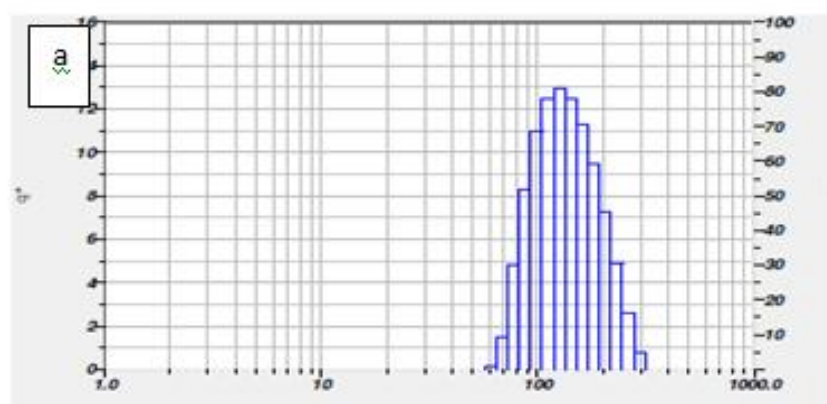

\section{Cumulant Operations}

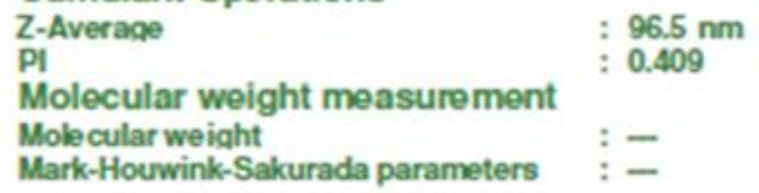

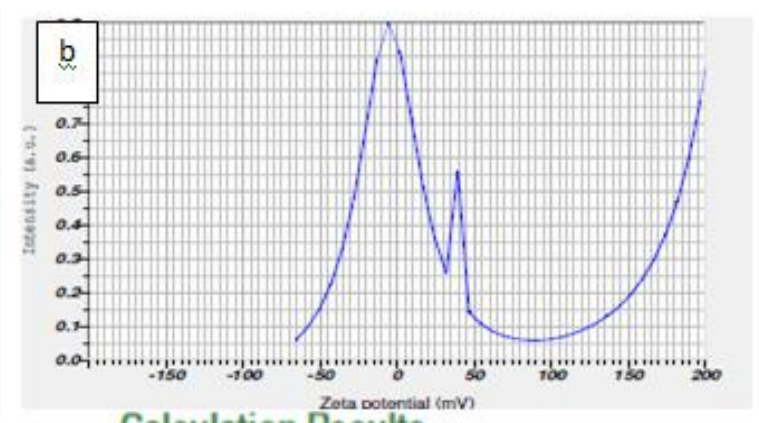

Calculation Results

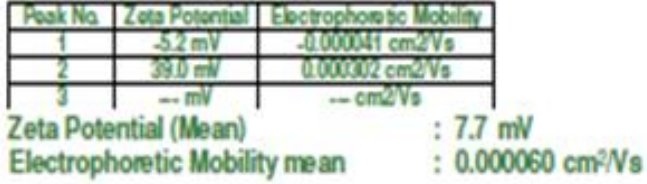


Fig.4 Showing SEM images of Gracilaria corticata mediated synthesis of silver nanoparticles
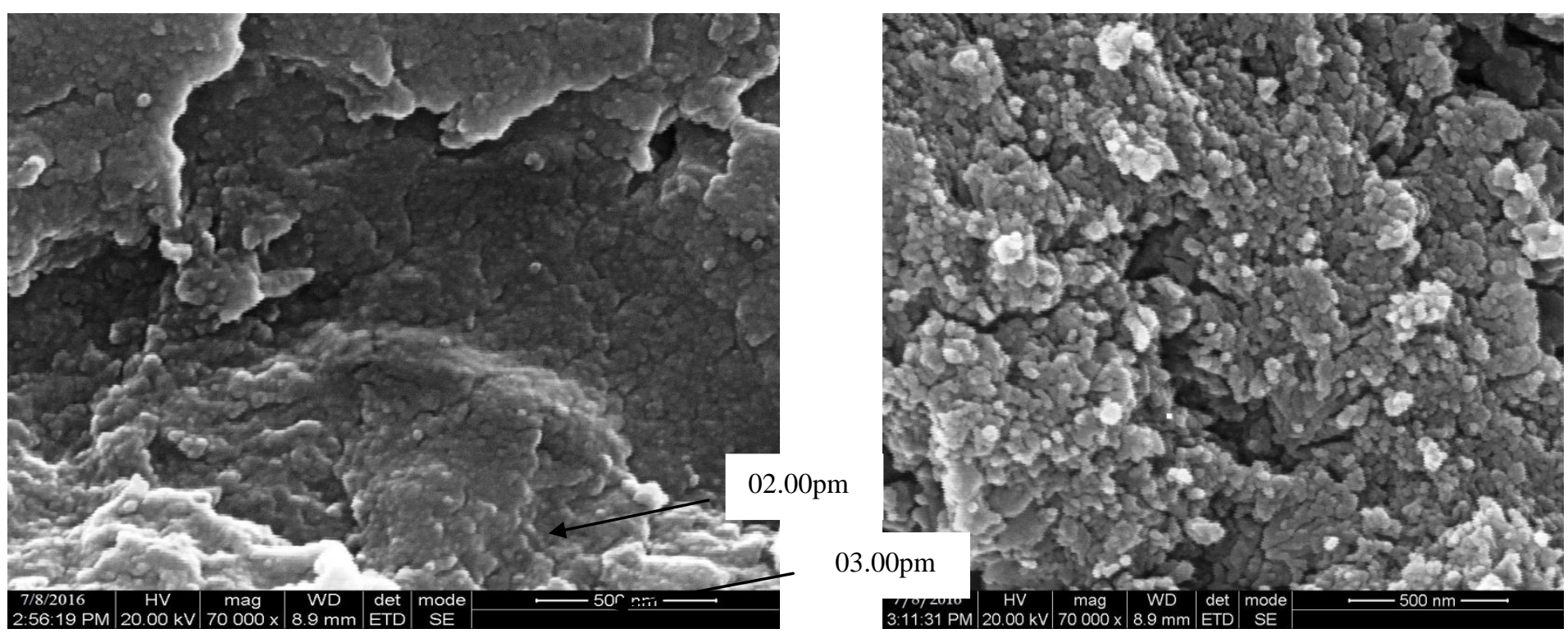

Fig.5 Showing Dye degradation of Malachite green by Gracilaria corticata mediated synthesis of silver nanoparticles

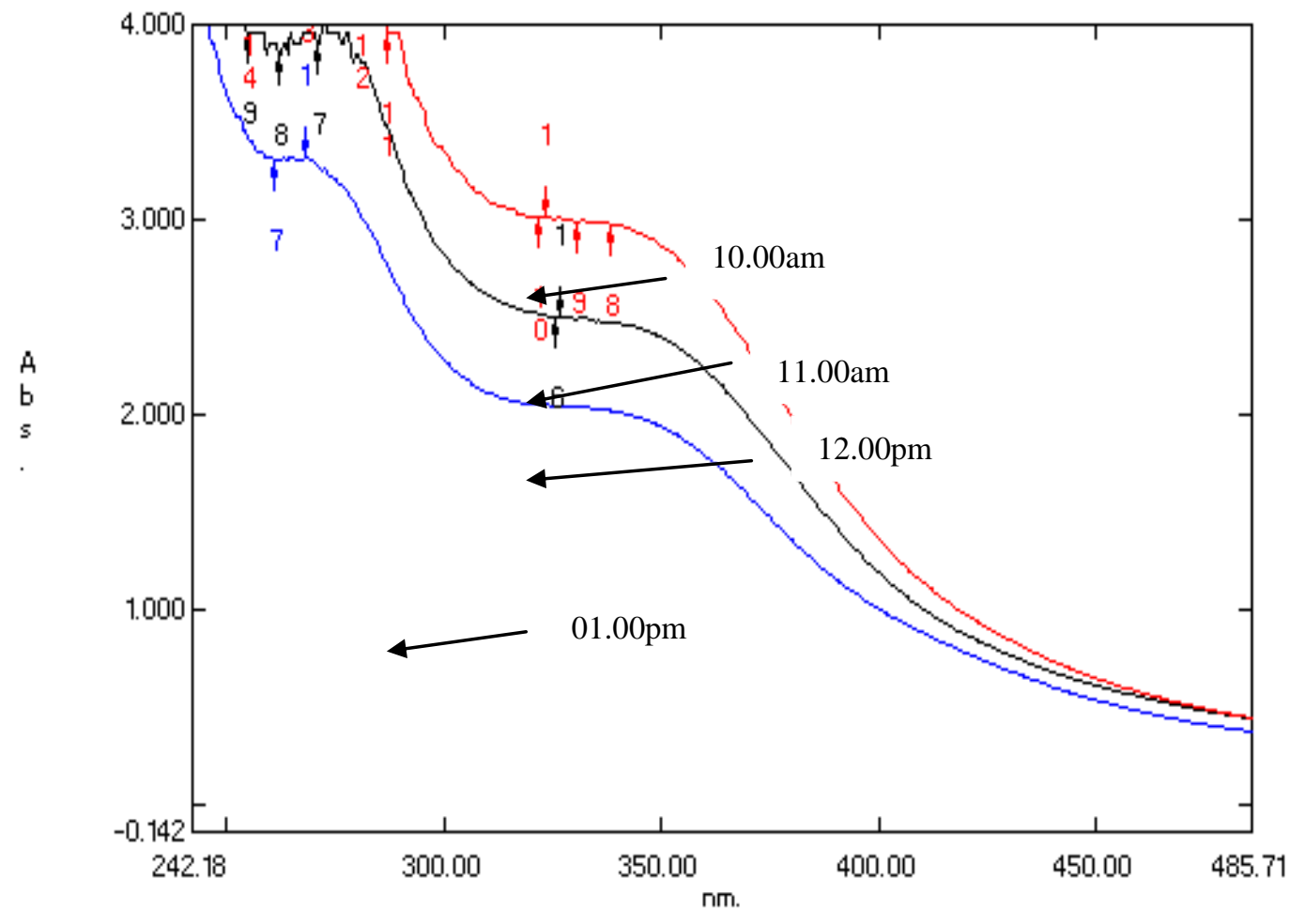




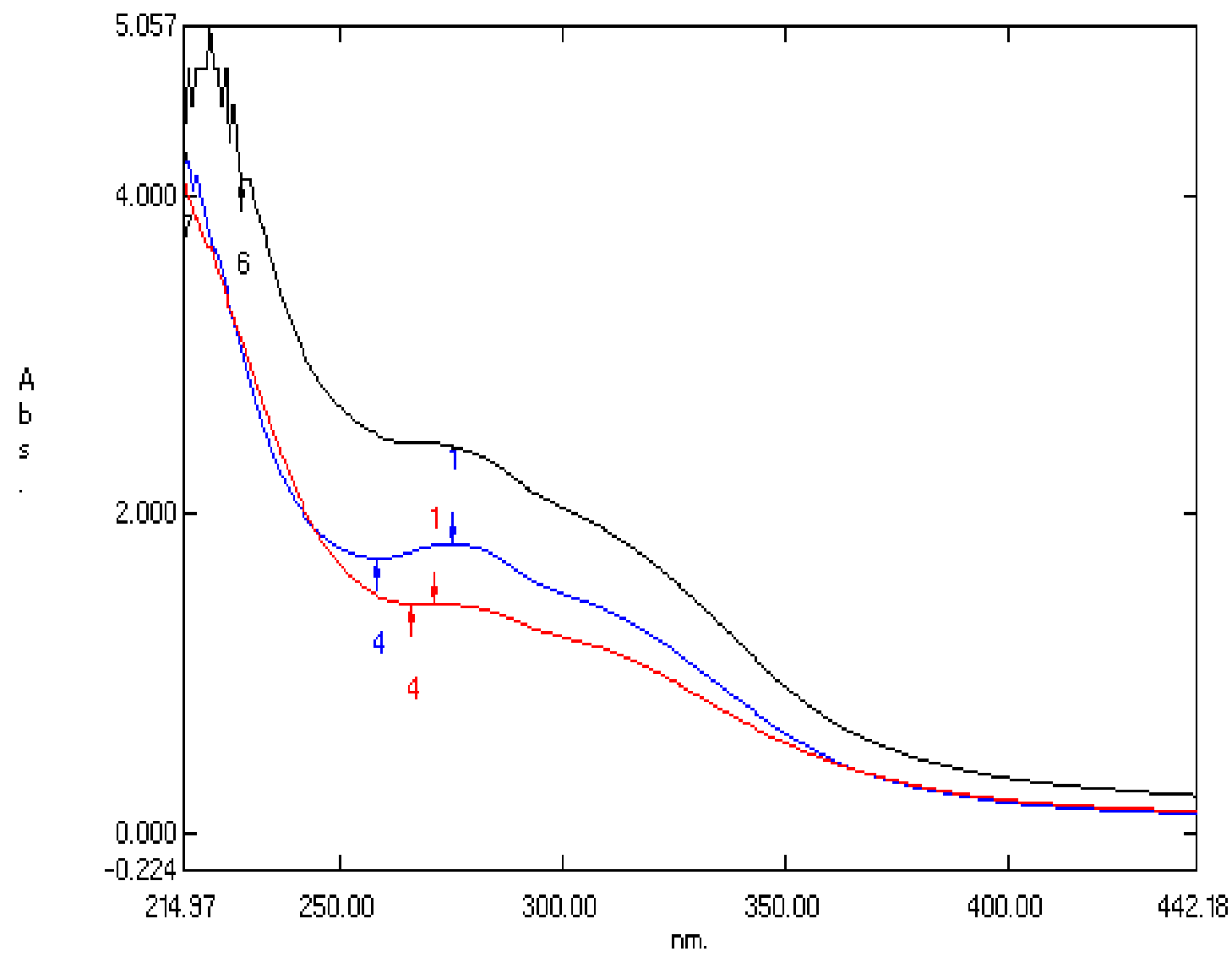

The adsorption of Ag nanoparticles on to the malachite green solution was initially low and further increased with constant increase in time. Altogether, the photo-catalytic properties of $\mathrm{Ag}$ nanoparticles in visible light may well be due to excitation of SPR, which is nothing but oscillation of charge density that can propagate at the interface between metal and dielectric medium. Guo et al., (2008) stated that, Ag nanoparticles are good, highly efficient and stable photo-catalysts under ambient temperature with visible light illumination for degrading organic compounds and dyes. The nanoparticles were found to be active in degrading malachite green solution with visible light illumination. These findings suggests that, silver nanoparticles synthesized by facile method from Gracilaria corticata can able to degrade dyes in the presence of visible light and paves way for environmental bioremediation very effective dye degradation towards malachite green.
The mechanism of sunlight irradiation strategy has been explained that the solar photons hit the nanoparticles present in the reaction mixture during exposure in sunlight, the electrons at the particle surface are excited and reacts with dissolved oxygen molecules in the reacting medium and converted into oxygen anion radicals. These radicals break the organic dye into simpler organic molecules leading to the rapid degradation of the dye. Therefore, the phyto-synthesized silver nanoparticles may act as a stable and efficient photo-catalyst for degradation of malachite green under visible light irradiation

In general, the greater the amount of the photo-catalyst, the higher rate of reaction due to increased active sites of the photo-catalyst would be. However, at the same time more photo-catalyst would also have induced greater aggregation, which resulted in the decrease in the surface area of the photocatalyst, making a significant fraction of the 
catalyst to be inaccessible to adsorbing the dye. Besides this, the degradation rate constant decreased after an optimum value for the photo-catalyst loading as in the concentration of dye, the opacity of dye solution also increased and UV radiation got scattered reducing the optical path. In these conditions, the penetration depth of the photon was decreased and fewer catalyst nanoparticles could be activated.

Degradation of methyl orange was visualized by decrease in peak intensity within $6 \mathrm{~h}$ of incubation time. There is no considerable shift in peak position for malachite green solution without exposure to Ag nano-catalysts.

In conclusion, silver nanoparticles from Gracilaria corticata were synthesized biometrically in room temperature within $24 \mathrm{~h}$ of incubation time. The formation of silver nanoparticles was confirmed by UV spectra, FE-SEM, and zeta potential measurements studies. The nanoparticles were found to be active in degrading malachite green solution with visible light illumination. These findings suggests that, silver nanoparticles synthesized by facile method from Gracilaria corticata can able to degrade dyes in the presence of visible light and paves way for environmental bioremediation.

\section{References}

Choi, O., Deng, K.K., Kim, N.J., Ross, L., Surampalli, R.Y., Hu, Z. 2008. The inhibitory effects of silver nanoparticles, silver ions, and silver chloride colloids on microbial growth. Water Res., 42(12): 3066-3074.

Dogan Uluozlu, O., Sari, A., Tuzen, M., Soylak, M. 2008. Biosorption of $\mathrm{Pb}$ (II) and $\mathrm{Cr}$ (III) from Aqueous Solution Bylichen. Parmelina tiliaceae) Biomass. Biores. Technol., 99: 2972-2880.

Fernandez, E.J., Garcia-Barrasa, J., Laguna,
A., Lopez-De-Luzuriaga, J.M., Monge, M., Torres, C. 2008. The preparation of highly active antimicrobial silver nanoparticles by an organ metallic approach. Nanotechnol., 19(18): Article ID 185602.

Guo, J.Z., Cui, H., Zhou, W., Wang, W. 2008. $\mathrm{Ag}$ nanoparticle-catalyzed chemiluminescence reaction between luminol and hydrogen peroxide. $J$. Photochem. Photobiol. A., 193(2-3): 89-96.

Hojberg, O., Canibe, N., Poulsen, H.D., Hedemann, M.S., Jensen, B.B. 2005. Influence of dietary zinc oxide and copper sulfate on the gastrointestinal ecosystem in newly weaned piglets. Appl. Environ. Microbiol., 71(5): 22672277.

Ismail, A.A. 2012. Facile Synthesis of Mesoporous Ag-Loaded $\mathrm{TiO}_{2}$ Thin Film and Its Photocatalytic Properties. Microporous and Mesoporous Materials, 149: 69-75.

Kansal, S.K., Singh, M., Sud, D. 2008. Studies on $\mathrm{TiO}_{2} / \mathrm{ZnO}$ photo-catalyzed degradation of lignin. J. Hazardous Materials, 153(1-2): 412-417

Kaviya, S., Santhana lakshimi, J., Viswanathan, B. 2012. Biosynthesis of silver nano-flakes by Crossandra infundibuliformis leaf extract. J. Mater. Lett., 67: 64-6.

Kokura, S., Handa, O., Takagi, T., Ishikawa, T., Naito, Y., Yoshikawa, T. 2010. Silver nanoparticles as a safe preservative for use in cosmetics. Nanomed., 6(4): 570-574, 2010.

Li, Y., Xiaodong, L., Junwen, L., Jing, Y. 2005. Photo-catalytic degradation of methyl orange in a sparged tube reactor with $\mathrm{TiO}_{2}$-coated activated carbon composites. Catalysis Communications, 6: 650-655.

Papinutti, L., Mouso, N., Forchiassin, F. 2006. Removal and Degradation of the 
Fungicide Dye Malachite Green from Aqueous Solution Using the System Wheat Bran-Fomes Sclerodermeus. Enzyme and Microbial Technol., 39: 848-853.

Roe, D., Karandikar, B., Bonn-Savage, N., Gibbins, B., Roullet, J.B. 2008. Antimicrobial surface functionalization of plastic catheters by silver nanoparticles. J. Antimicrob.
Chemother., 61(4): 869-876.

Salata, O.V. 2004. Applications of nanoparticles in biology and medicine. J. Nanobiotechnol., 2: 3-8.

Saxena, A., Tripathi, R.M., Zafar, F., Singh, P. 2012. Green synthesis of silver nanoparticles using aqueous solution of Ficus benghalensis Leaf extract and characterization of their antibacterial activity. J. Mater. Lett., 67: 91-4.

\section{How to cite this article:}

Subramanian Poornima and Karuppiah Valivittan. 2017. Degradation of Malachite Green (Dye) by using Photo-Catalytic Biogenic Silver Nanoparticles Synthesized using Red Algae (Gracilaria corticata) Aqueous Extract. Int.J.Curr.Microbiol.App.Sci. 6(1): 62-70. doi: http://dx.doi.org/10.20546/ijcmas.2017.601.008 